\title{
Diagnóstico e intervenção precoce no autismo: relatos de práticas profissionais
}

\author{
Diagnosis and early intervention in autism: reports of professional practices
}

\author{
Helen Aparecida Esteves', Jéssica Lorena de Morais², Jéssica Pâmela Alves da Silva \\ Santana $^{3}$ e Acríssio Luiz Gonçalves ${ }^{4}$
}

\begin{abstract}
Resumo: O Transtorno do Espectro Autista é caracterizado por déficits ou prejuízos persistentes na comunicação e na interação social, comumente aliados a padrões restritos e repetitivos de comportamento, interesses ou atividades. Oobjetivo da presente pesquisa foianalisar re relato de práticas de profissionais psicólogos que atuam em casos de autismo. Os dados foram coletados por meio de entrevistas semiestruturadas com nove psicólogas(os) atuantes em Minas Gerais e que realizam atendimento a crianças de 0 a 4 anos com risco e/ou diagnóstico de autismo, a partir da perspectiva da Análise do Comportamento Aplicada. Em geral, as(os) psicólogas(os) entrevistadas(as) apontaram a importância de compreender adequadamente o desenvolvimento infantil para que seja possível o rastreamento dos sinais de risco para o diagnóstico de autismo. Os(as) profissionais entrevistados (as) também destacaram que uma intervenção bem-sucedida requer o estabelecimento de um plano de trabalho que considere as especificidades de cada caso e que incentive os familiares na adoção de uma postura ativa no tratamento.
\end{abstract}

Palavras-chave: Transtorno do espectro autista; Intervenção precoce; Análise do comportamento aplicada

\begin{abstract}
The Autistic Spectrum Disorder is characterized by persistent losses or deficits in communication and social interaction, commonly combined with restricted and repetitive patterns of behavior, interests or activities. The aim of the study was to analyze the reports of professional practices of psychologists who work in cases of autism. Data were collected through semi-structured interviews with nine psychologists acting in Minas Gerais and who care for children from 0 to 4 years with risk or diagnosis of autism, from the perspective of Behavior Analysis Applied. In general, the psychologists pointed out the importance to understand properly the child development so that it is possible to enable tracking of signs of risk for the diagnosis of autism. The professionals interviewed also pointed out that a successful intervention requires the establishment of a work plan that considers the specificities of each case and that encourages family members in adopting an active approach to treatment.
\end{abstract}

Keywords: Autism spectrum disorder; Early intervention; Applied behavior analysis

\footnotetext{
1 Psicóloga pelo Centro Universitário Una. Pós-graduanda em Terapia Cognitivo Comportamental no Child Behavior Institute of Miami. E-mail: he.esteves@hotmail.com

2 Psicóloga pelo Centro Universitário Una. Cursa MBA em Gestão de Pessoas: Carreiras, Liderança e Coaching pela Pontifícia Universidade Católica do Rio Grande do Sul (PUCRS). E-mail: jessicalorena.morais@gmail.com

3 Psicóloga pelo Centro Universitário Una. Pós-graduanda em Intervenção ABA para Autismo e Deficiência Intelectual e em Desenvolvimento Infantil no Child Behavior Institute of Miami. E-mail: psicojessicasantana@gmail.com

4 Psicólogo. Doutor em Filosofia pela Universidade Federal de Minas Gerais (UFMG). Professor do Centro Universitário Una. E-mail:acrissio@yahoo.com.br
} 


\section{Introdução}

0 Transtorno do Espectro Autista (TEA) é caracterizado por déficits ou prejuízos persistentes na comunicação e na interação social, frequentemente aliados a padrões estereotipados, restritos ou repetitivos de comportamento, interesses ou atividades (American Psychiatric Association, 2014). No entanto, é possível identificar grande variação com relação ao nível de gravidade e comprometimento funcional relacionado ao transtorno, por isso o uso de termo"espectro".

0 TEA tem origem na infância e, na maioria dos casos, os sinais do transtorno podem ser consistentemente identificados entre os 12 e 24 meses de idade - ou mesmo antes disso, a depender da gravidade dos atrasos do desenvolvimento (Mesquita \& Pegoraro, 2013; Sociedade Brasileira de Pediatria, 2019). Essa identificação precoce dos sinais de risco para o autismo é importante porque permite a antecipação da intervenção, ampliando as possibilidades de desenvolvimento da criança e minimizando possíveis comprometimentos futuros (Murari \& Micheletto, 2015). Contudo, no Brasil, ainda há crianças que mantêm seu diagnóstico em aberto até os seis ou sete anos de idade (Silva \& Mulick, 2009).

A Análise do Comportamento Aplicada (ABA, do inglês Applied Behavioral Analysis) tem contribuído para a intervenção precoce no TEA e propiciado uma melhora significativa na qualidade de vida destes pacientes (Brasil, 2015). Em síntese, a ABA pode ser descrita como uma ramo tecnológico da Análise do Comportamento (Neto, 2002), cujas intervenções partem da identificação da função que os comportamentos adquirem na relação organismo-ambiente, 0 que permite o levantamento das possíveis variáveis comportamentais relevantes para o planejamento do tratamento clínico (Banaco, Zamignani, Martone \& Vermes, 2015; Meyer, Leonardi \& Oshiro, 2018; Sella \& Ribeiro, 2018). Nos casos de TEA, a ABA prioriza a criação de estratégias de intervenção que visam desenvolver áreas com déficits previamente detectados, de modo a promover maior bem-estar ao sujeito e seus familiares.

0 Modelo Denver de Intervenção Precoce (ESDM, do inglês Early Start Denver Model) também tem contribuído de forma eficaz em intervenções precoces de crianças com risco de TEA (Warren, 2011). As intervençōes a partir deste modelo abordam de forma precoce as dificuldades identificadas no desenvolvimento da criança, a partir de intervenções terapêuticas naturalísticas que auxiliam no desenvolvimento de habilidades diversas. Nessas intervenções, têm-se como objetivo a construção das interações sociais, relações de afeto e espontaneidade (Rogers \& Dawson, 2010). Tal modelo de intervenção concilia a abordagem comportamental com a perspectiva desenvolvimentista.

Ambas as propostas de intervençōes supracitadas, a ABA e o ESDM, podem ser usadas de forma individual ou em conjunto na proposição de estratégias de intervenção em casos de TEA. Nessa perspectiva, o presente estudo teve como objetivo evidenciar a aplicabilidade da ABA na prática clínica em crianças de 0 a 4 anos com risco ou já diagnosticadas com TEA, a partir do relato das práticas de profissionais psicólogas(os) que atuam em tais casos. Para tal, realizou-se entrevistas junto a profissionais formados em Psicologia que direcionam 0 atendimento a este público, a partir da ABA, no estado de Minas Gerais.

\section{Método}

A presente pesquisa trata-se de um estudo de campo de caráter exploratório, tendo como enfoque o de descrever a intervenção psicoterapêutica em crianças de 0 a 4 anos com risco (ou com diagnóstico) de TEA. Participaram dessa pesquisa 09 (nove) profissionais psicólogas(os) que atuam no estado de Minas Gerais e possuem, como base de intervenção, a ABA e/ou o ESDM, sendo esta amostra estabelecida por conveniência.

Para coleta de dados foi utilizada uma entrevista semiestruturada, a partir de um roteiro composto por 12 perguntas. Em sua maioria, as entrevistas foram realizadas presencialmente, no consultório dos profissionais entrevistados, conforme solicitação dos mesmos; entretanto, alguns profissionais encaminharam as respostas via e-mail. As entrevistas foram transcritas e, em seguidas, analisadas a partir de diversas categorias, considerando as principais perguntas do roteiro de entrevista.

Este estudo seguiu as resoluções 466/12 e 251/97 referentes ao Conselho Nacional de Saúde (CNS). Os participantes assinaram o Termo de Consentimento Livre e Esclarecido (TCLE) e a pesquisa foi devidamente submetida à Plataforma Brasil e aprovada por um Comitê de Ética em Pesquisa, a partir do parecer número 3.282.501. Os dados dos participantes entrevistados são apresentados na Tabela 1.

Tabela 1: Descrição das Participantes da Pesquisa

\begin{tabular}{|c|c|l|}
\hline Participante, idade, sexo & Ano de graduação em psicologia & $\begin{array}{l}\text { Percurso formativo após a graduação } \\
\text { em psicologia }\end{array}$ \\
\hline$P 1,34, F$ & 2007 & $\begin{array}{l}\text { Formação em Terapias Cognitivas, Especialização } \\
\text { em Terapia Comportamental, Mestrado e Douto- } \\
\text { rado em Desenvolvimento Humano. }\end{array}$ \\
\hline$P 2,27, \mathrm{M}$ & 2015 & $\begin{array}{l}\text { Cursando Especialização em Psicologia Compor- } \\
\text { tamental. }\end{array}$ \\
\hline$P 3,33, \mathrm{~F}$ & 2018 & Não possui. \\
\hline$P 4,24, \mathrm{~F}$ & 2017 & $\begin{array}{l}\text { Cursando Especialização em Psicologia Compor- } \\
\text { tamental. }\end{array}$ \\
\hline$P 5,40, \mathrm{~F}$ & $\begin{array}{l}\text { Especialização em Educação Especial Inclusiva e } \\
\text { em Psicologia Comportamental. }\end{array}$ \\
\hline
\end{tabular}




\begin{tabular}{|c|c|l|}
\hline Participante, idade, sexo & Ano de graduação em psicologia & $\begin{array}{c}\text { Percurso formativo após a graduação } \\
\text { em psicologia }\end{array}$ \\
\hline$P 6,24, \mathrm{M}$ & 2018 & Não possui. \\
\hline$P 7,28, \mathrm{~F}$ & 2013 & $\begin{array}{l}\text { Especialização em ABA e Mestrado em Neuro- } \\
\text { ciência. }\end{array}$ \\
\hline $\mathrm{P} 8,27, \mathrm{~F}$ & 2014 & $\begin{array}{l}\text { Formação em Terapias de Terceira Onda, Especia- } \\
\text { lização em Terapia Cognitivo-Comportamental e } \\
\text { Mestrado em Neurociência. }\end{array}$ \\
\hline $\mathrm{P9}, 26, \mathrm{~F}$ & $\begin{array}{l}\text { Formação em Terapia por Contingência de Refor- } \\
\text { çamento; Cursando Especialização em Psicologia } \\
\text { Comportamental. }\end{array}$ \\
\hline
\end{tabular}

\section{Resultados}

Os relatos dos(as) psicólogos(as) foram agrupados em quatro macro categorias, de modo a enfatizar as respostas de maior relevância para a pesquisa, sendo elas: 1) "Sinais de riscos para o TEA"; 2) "Intervenção precoce em casos de TEA"; e 3) "Relevância da Análise do Comportamento Aplicada nas intervenções em casos de TEA". Também foram elegidas subcategorias de análise, com base nas especificidades do roteiro de entrevista utilizado.

0 Quadro 1, a seguir, apresenta al guns relatos dos participantes relativos à categoria 1, sobre os sinais de risco para o TEA.

Quadro 1 - Categoria 1: Sinais de risco para o TEA

\section{Relatos das(os) psicólogas(os) entrevistadas(os)}

"Ao contrário de crianças neurotípicas, o desenvolvimento de crianças dentro do TEA foge de certos critérios esperados para os primeiros anos de vida. Dentre eles, a falta ou o pouco contato visual, atraso global na comunicação verbal e não-verbal, interesses restritos e/ou estereotipados e uma hiper ou hiposensibilidade sensorial". (P6)

"Cada vez mais estamos avançando no diagnóstico precoce de TEA e os sinais para um possível diagnóstico variam bastante de criança para criança. Entretanto, é comum as queixas dos pais sobre a criança não atender ao nome quando chamada, não manter contato visual como esperado, ter dificuldades de interação e apresentar comportamentos estereotipados". (P4)

"Eu faço um histórico bem detalhado desde à gravidez. Procuro relembrar os pais de todos os detalhes possíveis do desenvolvimento. Fico muito atenta para checar sempre os sinais e sintomas mais típicos (apego à rotina, dificuldades na comunicação, enfileirar objetos ou brinquedos, apego a objetos, brincar não funcional, estereotipias etc.), e atenta para entender também como está ocorrendo o desenvolvimento. Se está contínuo, se tem saltos inexplicáveis, se estacionou em alguma etapa". (P5)

Os resultados referentes à Categoria 2," Intervenção precoce em casos de TEA", foram subdivididos em cinco subcategorias, a saber: 2.1) Importância da intervenção precoce; 2.2) Planejamento das intervenções; 2.3) Consolidação das intervenções; 2.4) Importância do treinamento de pais; e 2.5) Desafios do atendimento a crianças com TEA.

0 quadro 2, a seguir, apresenta alguns dos relatos sobre a importância da intervenção precoce em casos de TEA (subcategoria 2.1).

Quadro 2 - Subcategoria 2.1: Importância da intervenção precoce

\section{Relatos das(os) psicólogas(os) entrevistadas(os)}

"A importância é a de antecipar um efeito cascata de danos, evitando expor a criança e a família a uma sequência de insucessos. Além de não melhorar, a tendência da criança sem intervenção é piorar, e a família se frustra muito. Então, muitas vezes, quando atendemos uma família com uma criança mais velha, a família já está muito cansada e a criança muito esquiva pela quantidade de experiências de insucesso. Então, intervir cedo evita esse monte de repercussões - inclusive, psicológicas - para os pais e para criança também". (P1)

"A intervenção precoce é fundamental no desenvolvimento de uma criança com TEA [...]. Quanto mais tempo se demora para fazer uma intervenção, maior éa probabilidade de 0 atraso aumentar. Com o passar do tempo esse atraso fica muito discrepante e pode se tornar crônico. Então, a intervenção precoce éfundamental para que a criança possa se desenvolver e, em dado momento, possa ter uma certa independência ou até mesmo a total independência dos familiares". (P9) 
"Os sintomas iniciais do autismo privam essas crianças de acessar, no nível das [crianças] neurotípicas, as mesmas oportunidades de interação e aprendizagem social. Enquanto bebês e crianças pequenas, tais diferenças podem ser sutis ou moderadas, mas na falta de uma intervenção adequada, ocorre um efeito cascata e a diferennça se torna deficiência. Na ausência de habilidades comunicativas e sociais básicas, fica cada vez mais difícil para essas pessoas conseguirem acompanhar o ritmo e a complexidade crescente das demandas sociais em família, na escola e, futuramente, nos relacionamentos e no trabalho. Há, então, um comprometimento crônico para a independência e qualidade de vida. Além disso, existe maior plasticidade neural até os 5 anos de idade, faixa etária em que a criança está mais apta para aprender de modo intensivo e, por consequência, perído em que as intervenções possuem maior valor terapêutico". (P6)

0 quadro 3, a seguir, apresenta alguns relatos das(os) psicólogas(0s) zação das intervenções (subcategoria 2.2). entrevistadas(os) que exemplificam o processo de planejamento e a organi-

Quadro 3 - Subcategoria 2.2: 0 planejamento das intervenções

\section{Relatos das(os) psicólogas(os) entrevistadas(os)}

"Você vai escolhendo as brincadeiras de acordo com o interesse de cada criança, aí você vai trabalhando as metas. Em alguns momentos, têm crianças que não gostam de nada, esse é o maior desafio. Então, é preciso começar com alguma coisa muito básica e usar o pareamento de estímulos. Parear uma coisa muito básica que ela goste com alguns brinquedos que são neutros, para aqueles brinquedos irem ficando, de certa forma agradáveis e, assim, ampliar o repertório [de estímulos reforçadores] da criança". (P7)

"Eu uso, por exemplo, um jogo vai e vem. É um tipo de jogo que uso para fazer perguntas. Então, escrevo qual é o objetivo: fazer perguntas, responder perguntas, manter interação, por exemplo. Eaí, seguindo essa planilha, vou implementando aquilo que quero trabalhar com cada atividade". (P2)

"Eu mesclo, por habilidade a ser desenvolvida, habilidades mais fáceis com habilidades mais difíceis, e à medida que a criança consegue realizar a habilidade " $x$ " sem nenhum tipo de ajuda, em no mínimo três intervenções consecutivas, eu entendo que ela já adquiriu aquela habilidade. Apesar de eu passar para outra habilidade, esta que ele já adquiriu eu continuo reforçando de modo a auxiliar na generalização de brincadeiras, habilidades, objetos etc.". (Р3)

O quadro 4, a seguir, apresenta alguns relatos dos participantes sobrea conso- lidação das aprendizagens elegidas como metas de intervenção (subcategoria 2.3).

Quadro 4 - Subcategoria 2.3: Consolidação das intervenções

\section{Relatos das(os) psicólogas(os) entrevistadas(os)}

"Quando temos uma [alta] frequência. Por exemplo, você dá dez oportunidades para criança e, em mais de sete oportunidades, a criança apresenta de forma espontânea aquele comportamento, consideramos que o comportamento está consolidado. Então, fazemos isso com todos os comportamentos e, assim, passamos para o nível seguinte. A ideia é a de que o comportamento consolidado aconteça em mais de 70\% das oportunidades e, ao mesmo tempo, continuamos observando se ele vai diminuir, se temos que continuar com a intervenção". (P8)

“Eu só considero que a criança adquiriu aquela meta se ela conseguiu executar três dias consecutivos aquela meta, com total independência. E a independência depende que ela realize a meta que planejei sem o suporte. A conclusão desta meta se dá depois três dias, mas normalmente a aprendizagem dura cerca de quatro meses. Então, após quatro meses, a gente faz a reavaliação e modifica todas as metas elaboradas para aquela criança”.". (P9)

"Temos alguns parâmetros para dizer que a criança realmente consolidou a habilidade. Um parâmetro é a generalização entre locais, outro parâmetro é a generalização entre pessoas e um terceiro parâmetro é quando o comportamento não se encontra tão suscetível a fatores motivacionais. Pois, se uma habilidade está sempre pareada com reforçador, será que o comportamento vai estar dependente do reforç̧o arbitrário? [...] A criança vai no banheiro e faz o "xixi" sozinha, com fluência, ou escova os dentes com fluência, aponta com fluência, então, isso quer dizer que tais comportamentos não estão mais dependentes, necessariamente, de um reforçador arbitrário. A criança já faz, como um hábito: às vezes ela tem feedback, às vezes ela não tem feedback, mas continua fazendo". (P1)

0 quadro 5 apresenta relatos dos participantes que ressaltam a importância do treinamento de pais (subcategoria 2.4), auxilio indispensável no tratamento de crianças com TEA. 
Quadro 5 - Subcategoria 2.4: A importância do Treinamento de Pais

\section{Relatos das(os) psicólogas(os) entrevistadas(os)}

"Acho que [a participação dos pais] é uma das coisas mais importantes. Alguns pais que não têm condição financeira; então, às vezes eu prefiro até reduzir as sessões com a criança e aumentar 0 treinamento de pais, porque são eles que ficam a maior parte do tempo com a criança, então, 0 desenvolvimento maior é com eles. É claro que a nossa intervenção é importante também, mas acho fundamental [...] que eles possam continuar estimulando a criança em casa". (P2)

"A relevância é enorme. Tendo em vista que geralmente o número de horas que os pacientes passam em terapia é pequeno, a continuidade dessas atividades durante o dia e a semana só é possível se os pais/cuidadores estiverem informados e habilitados para complementarem o tratamento". (P5)

"A principal característica do treinamento de pais é trabalhar a generalização. Então, os pais sabem o que fazer e exigem um determinado comportamento que está no plano de tratamento, de modo que a criança consiga usar isso em todos os contextos". (P7)

O quadro 6 apresenta alguns relatos acerca dos desafios vivenciados ao longo do atendimento de crianças com risco e/ou diagnóstico de TEA (subcategoria 2.5).

Quadro 6 - Categoria 2.5: Desafios do atendimento a crianças com TEA

\section{Relatos das(os) psicólogas(os) entrevistadas(os)}

“Não é um trabalho acessível, porque envolve muitos profissionais: envolve fonoaudiólogo, terapeuta ocupacional, musicoterapeuta, psicólogo, educador físico, a escola e, muitas vezes, a mediação domiciliar. É um trabalho caro, um tratamento caro. Então, uma das principais dificuldades é a acessibilidade ao tratamento". (P9)

"Vejo o estado emocional dos pais como um dificultador no início. Como estão muito abalados, muitas vezes atrapalham nosso trabalho, especialmente com tantas informações bizarras na internet, as promessas de curas etc. Dá trabalho trazê-los de volta à realidade. Também sinto muita dificuldade na relação com a escola". (P5)

"Em termos de dificultadores, eu acho que são vários. Por exemplo, um pediatra pode ter dificuldade para diagnosticar, a família pode ter dificuldade em aceitar, inclusive, o custo do tratamento - porque, na minha opinião, o custo é um grande problema. No país que a gente vive, o preço de se tratar uma criança com TEA é muito alto. Eas pessoas ainda naturalizam muito as dificuldades no desenvolvimento, dizendo que cada um tem seu tempo. Às vezes as pessoas ficam com medo de alarmar os pais [...]; ao contrário, eu sempre falo com os pais: eu prefiro pecar pelo excesso. Digo que existe um atraso no desenvolvimento e que é preciso trabalhar, assim ninguém vai perder com isso". (P8)

0 quadro 7 apresenta os dados relacionados à terceira categoria de análise, que trata da importância da ABA na intervenção precoce em crianças com risco ou diagnóstico de TEA. Além disso, aborda questões relativas à formação

específica - do ponto de vista teórico, técnico e ético - para a prestação de um serviço de qualidade.

Quadro 7 - Categoria 3: Relevância da Análise do Comportamento Aplicada nas intervenções em casos de TEA

\section{Relatos das(os) psicólogas(os) entrevistadas(os)}

"A Análise do Comportamento fornece os princípios da aprendizagem: como as pessoas aprendem determinados comportamentos e como manejamos o aumento da frequência de emissão de determinado comportamento ou a reducãao da emissão de determinado comportamento. Um ponto positivo da Análise do Comportamento é a simplicidade da análise funcional, que condensa tão bem o funcionamento comportamental tanto de crianças quanto de adultos. Então, é uma abordagem muito parcimoniosa e objetiva. Assim, você tem que mapear os antecedentes e as consequências dos comportamentos; não tem nenhum segredo, então, isso eu acho um ponto muito positivo. Um ponto que eu acho muito negativo na Análise do Comportamento é o número de termos técnicos que, às vezes, distanciam o profissional da família. Outro ponto negativo é que, na medida em na Análise do Comportamento ésimples e parcimoniosa, alguns profissionais fazem um uso artificial demais, simplificado demais, o que dificulta a generalização [da aprendizagem]. Mas, eu não acho que é um crítica à abordagem em si, e sim uma crítica ao mal-uso da abordagem". (P1)

"Hoje eu não consigo imaginar outra abordagem que não a Análise do Comportamento ou a TCC [Terapia Cognitivo-Comportamental] para as intervenções com autismo. A Análise do Comportamento permite ensinar novas habilidades para a criança, para que ela tenha autonomia e qualidade de vida. Em relação aos pontos positivos e negativos, acredito que o que determina é o profissional, pois, se ele tem clareza do transtorno, se entende o contexto em que a criança está inserida e trabalha metas realistas que vão fazer sentido para a criança e a família, todos têm a ganhar. Porém, infelizmente, existem muitos profissionais que não desempenham o trabalho de maneira ética e isso faz com a que, muitas vezes, a abordagem seja malvista - por exemplo, muitos pais têm o discurso de que a Análise do Comportamento faz com que a criança fique robotizada. Realmente, se eu não consigo variar a brincadeira com criança e realizo o treino no sentido de que cada vez que a criança faz "x" ela ganha "y", ela ficará robotizada mesmo, mas não porque a abordagem é assim, e sim porque o profissional talvez não tenha entendido o seu papel diante da criança, diante da família e do próprio transtorno". (P3) 
"As intervenções baseadas em Análise do Comportamento são as que mais apresentam evidências científicas no tratamento do Autismo, o que a torna mais viável do que uma série de outras abordagens para essa demanda. Entretanto, há profissionais que não se embasam o suficiente teoricamente e acabam reproduzindo os conceitos da Análise do Comportamento e suas técnicas de maneira equivocada". (P4)

\section{Discussão}

No que se refere à Categoria 1, "Sinais de riscos para oTEA", os profissionais ressaltaram alguns indicadores que podem ser observados desde os primeiros anos de vida, tais como baixo contato ocular, déficit na atenção compartilhada, baixa frequência de sorriso e reciprocidade social, pouca participação em brincadeiras de forma funcional, dificuldade de imitação motora e atraso ou ausência na comunicação verbal ou não verbal. Desse modo, alterações nos âmbitos da linguagem, interação social e comportamentos repetitivos têm sido apontados como sinais precoces de TEA, servindo como parâmetros para 0 início da intervenção (Sociedade Brasileira de Pediatria, 2019).

Oito profissionais consideram que intervir precocemente é importante para o processo de desenvolvimento da criança, enquanto um participante (P6) não respondeu diretamente à pergunta, embora tenha ressaltando 0 valor terapêutico do tratamento. Os relatos coincidem com as orientações da Diretriz de Atenção à Reabilitação da Pessoa com TEA, publicada pelo Ministério da Saúde em 2014, documento que ressalta a importância da identificação precoce do TEA em crianças de até três anos de idade (Brasil, 2014). Além disso, é consenso na literatura especializada que, quanto mais precoce a intervenção, melhores são os resultados do tratamento, o que repercute em maior qualidade de vida para a criança diagnosticada com TEA (Gomes et al., 2015; Sociedade Brasileira de Pediatria, 2019).

As(os) psicólogas(os) mencionaram a necessidade de instrumentos de avaliação padronizados para identificar os sinais precoces de TEA. Em geral, tais instrumentais permitem a avaliação e identificação de risco de TEA na população em geral ou em crianças que já apresentam quadros indicativos de algum problema de desenvolvimento (Seize \& Borsa, 2017), seja por meio de informações fornecidas pelos pais e/ou cuidadores, seja a partir da própria observação clínica da criança. Os instrumentos listados pelos(as) entrevistadas(os) foram os seguintes: Autism Diagnostic Observation Schedule (ADOS); The Modified Checklist for Autism in Toddlers (M-CHAT);Verbal Behavior Milestones Assessment and Placement Program (VB-MAPP); e Checklist Curriculum do Modelo Denver de Intervenção Precoce para Crianças Pequenas com Autismo (Checklist Denver).

0 ADOS é um instrumento padronizado que permite o diagnóstico de TEA em crianças e adultos, a partir da observação do paciente em uma série de tarefas estruturadas que envolvem os domínios da comunicação e interação social (Lord et al., 1989). 0 ADOS foi concebido como um instrumento complementar ao Autism Diagnostic Interview-Revised (ADI-R), uma entrevista destinada a pais e/ou cuidadores que visa identificar os comprometimentos relativos ao autismo em diversas áreas do desenvolvimento (Lord et al., 1994). Ambos os instrumentos são apontados como "padrão-ouro" para 0 diagnóstico de TEA (Marques \& Bosa, 2015). À época do presente estudo não existia uma validação do ADOS para o contexto brasileiro; ainda assim, al guns entrevistados mencionaram se tratar de uma ferramenta de grande valia, uma vez que apresenta informações importantes para o processo de rastreio do TEA. Tal utilização, no entanto, levanta questões éticas que envolvem os direitos autorais e a ausência de adaptação e validação do referido instrumento. Recentemente, 0 instrumento recebeu uma versão traduzida e adaptada ao contexto brasileiro (Pacífico et al., 2019).
0 M-CHAT é instrumento de triagem que permite a identificação de sinais precoces de TEA em crianças entre 18 a 24 meses (Robins, et al., 2001), traduzido para o português brasileiro (Losapio \& Pondé, 2008) e validado para este contexto (Castro-Souza, 2011). 0 M-CHAT é citado como o instrumento para rastreio precoce de autismo mais utilizado em âmbito global (Hyman \& Johnson, 2012), além de ser o único instrumento internacional de uso livre adaptado e validado para o contexto brasileiro (Bosa \& Zanon, 2016). Atualmente, a Sociedade Brasileira de Pediatria (2019) recomenda ao uso da versão revisada desse instrumento, o M-CHAT-R/F (Robins, Fein, Barton \& Resegue, 2009), indicado para avaliação de risco de TEA em crianças de 16 a 30 meses de idade. A versão revisada é composta de 20 itens do tipo "sim/não" a serem respondidos pelos pais e/ou cuidadores da criança, e permite a identificação de baixo risco, risco moderado ou alto risco para o TEA, além de conter uma entrevista de follow-up que aumenta a sua eficácia (Seize \& Borsa, 2017).

O VB-MAPP é um instrumento que permite uma avaliação sistemática do repertório verbal e de outras habilidades relacionadas, considerando os marcos típicos do desenvolvimento em três faixas etárias: de 0 a 18 meses; de 18 a 30 meses; de 30 a 48 meses (Sundberg, 2014). Conforme ressaltam Ribeiro, Sella e Souza (2018), por se tratar de um instrumento elaborado com base nos operantes verbais descritos por B. F. Skinner (1957), o conhecimento destes conceitos é fundamental para que o aplicador consiga avaliar o nível do repertório verbal da criança. Instrumento traduzido e adaptado para 0 contexto brasileiro (Martone, 2017).

0 Checklist Denver é utilizado para avaliar bebês e crianças com TEA, considerando vários domínios do desenvolvimento, com intuito de permitir a estruturação do processo terapêutico. Organiza-se em quatro níveis de habilidades, considerando as seguintes faixas etárias: dos 12 aos 18 meses; dos 18 aos 24 meses; dos 24 aos 36 meses; dos 36 aos 48 meses (Rogers \& Dawson, 2010).

No Brasil, além dos instrumentos citados pelas(os) profissionais entrevistas(0s), existem outros instrumentos traduzidos, adaptados e parcialmente validados para a triagem de TEA, a saber: o Autism Behavior Checklist (ABC); o Autism Screening Questionnaire (ASQ); e a Childhood Autism Rating Scale (CARS) (Marques \& Bosa, 2015; Pacífico et al., 2019).

Antes do início do tratamento, o analista do comportamento deve realizar a avaliação comportamental da criança, de modo a obter uma visão panorâmica do quadro clínico, identificar os déficits existentes e realizar o plano de trabalho com a criança, sessão por sessão, com base em metas objetivamente identificadas e descritas (Ribeiro, Sella \& Souza, 2018). Assim, as sessões de intervenção são programadas com foco no reforçamento do comportamento ensinado à criança; são também individualizadas, isto é, planejadas considerando as especificidades de cada criança (Lafrance, 2018).

Seguindo tais princípios, as(os) profissionais entrevistadas(os) mencionaram que seus atendimentos devem ser realizados em um ambiente que melhor se adeque às necessidades de cada paciente, com o uso de brinquedos e objetos de interesse da criança, os quais são utilizados como estímulos reforçadores - uma atuação que depende da identificação dos estímulos que a criança prefere (comestíveis, brinquedos, atividades, entre outros), procedimento conhecido como avaliação de preferência (Ribeiro \& Sella, 2018). 
Em relação à consolidação das intervenções, dois profissionais utilizam como critério a generalização dos comportamentos para outros ambientes e com outras pessoas (P1, P6) e dois participantes avaliam a generalização por meio de três intervenções seguidas sem suporte $(\mathrm{P4}, \mathrm{P9})$. Uma entrevistada (P8) considera que a aprendizagem está consolidada quando a criança emite o comportamento ensinado em, pelo menos, 70\% das ocasiões esperadas. Apenas um participante não descreveu como identifica a consolidação da aprendizagem das metas (P4). Quanto à reavaliação dos objetivos, que tem como propósito o estabelecimento de novas metas e a verificação dos comportamentos ensinados, seis participantes (P1, P2, P5, P7, P8 e P9) utilizam como critério o tempo médio de 3 a 6 meses de intervalo. Vale ressaltar que alguns entrevistados utilizam dois ou mais critérios de avaliação dos repertórios comportamentais adquiridos.

Segundo a literatura especializada, os critérios de consolidação das intervenções variam de acordo com a natureza da atividade e o repertório comportamental da criança. Ainda assim, diversos autores apontam como critério a emissão do comportamento ensinado em alta frequência, nas ocasiões apropriadas - por exemplo, em 90\% ou em até 100\% das vezes, considerando um ou mais blocos de tentativas com a criança (Canovas, 2018; Almeida \& Martone, 2018). Após o al cance do critério de aprendizagem definido, o comportamento-alvo é colocado em manutenção, o que significa que deve ser monitorado periodicamente, de modo que a criança mantenha o seu desempenho ao longo do tempo, em diferentes contextos e com diferentes pessoas (Almeida \& Martone, 2018).

As(os) psicólogas(os) entrevistadas(os) apontaram aspectos que dificultam sua atuação, tais como: a demora na identificação de atrasos no desenvolvimento (P2, P3); a não aceitação do diagnóstico por parte dos pais e/ou familiares (P3, P5, P8, P9); o pouco envolvimento ou suporte da escola nas intervenções (P5); e 0 alto custo do tratamento (P2, P6, P8, P9). Ainda com relação ao al to custo do tratamento, um(a) dos profissionais (P2) relatou que costuma adequar 0 tratamento às condições financeiras da família, por exemplo, diminuindo o número de sessões semanais com a criança $e$ ampliando o número de sessões para o treinamento de pais.

De fato, a postergação diagnóstica do TEA é uma realidade no Brasil, onde 0 diagnóstico durante os anos pré-escolares ainda é bastante raro (Silva \& Mulick, 2009; Gomes et al., 2015). Ademais, os relatos das(os) profissionais entrevistadas(os) estão de acordo com a análise realizada por Barros, Barbosa e Silva (2018), que identifica o alto custo do tratamento, a pouca disponibilidade de profissionais devidamente capacitados e fragilidade das redes públicas de educação e de assistência à saúde como fatores que limitam o acesso de grande parte da população diagnosticada com TEA à intervenção precoce, intensiva e duradoura.

A dificuldade de aceitação do diagnóstico de TEA por parte da família, reportada por alguns dos entrevistados, pode ser influenciada por episódios de preconceito vivenciados pela criança em diversos ambientes, o que gera conflitos sociais e estresse familiar (Mapelli et al., 2018). Além disso, o estresse familiar pode ser agravado por diversos fatores, como a severidade do transtorno, a postergação diagnóstica, a dificuldade de acesso a serviços de saúde, a condição socioeconômica da família e a indisponibilidade de redes de apoio e suporte social (Andrade \& Teodoro, 2012; Gomes et al., 2015; Miele \& Amato, 2016). Esse cenário torna justificável a ampliação de intervenções centradas nos pais, uma estratégia importante para o bom andamento terapêutico e para a melhoria da qualidade de vida do grupo familiar.

No que concerne ao treinamento de pais, todos as(os) entrevistadas(os) relatam a importância dessa prática na generalização dos comportamentos adaptativos. Em geral, o treinamento capacita os pais a realizarem intervenções em casa, para que as lacunas comportamentais sejam melhor trabalhadas e para que as habilidades adquiridas pela criança sejam mais facilmente generalizáveis, isto é, se expressem em outros ambientes e contextos. As intervenções realizadas pelos pais e/ou cuidadores também são importantes para impedir eventuais declínios de habilidades e garantir o constante reaprendizado (Figueiredo, 2014). Além disso, a participação dos pais no gerenciamento de comportamentos dos filhos pode contribuir para a redução do estresse familiar, desde que os pais tenham acesso a treinamento especializado e 0 apoio necessário para lidar com as dificuldades relativas ao diagnóstico e ao tratamento do TEA (Mota, Vieira \& Nuernberg, 2020).

A importância da Análise do Comportamento no tratamento de crianças com diagnóstico de TEA é inquestionável nos relatos. Os pontos positivos dizem respeito ao respaldo científico da abordagem (P4, P5, P6, P7, P9), à eficácia das intervenções no ensino de novas habilidades (P3) eà possibilidade de realização de intervenções em contexto naturalístico (P8). Contudo, seis participantes (P2, P3, P4, P5, P7, P8, P9) relatam que a falta de conhecimento teórico e técnico em Análise do Comportamento pode trazer prejuízos ao cliente, com o estabelecimento de rotinas de intervenção pouco sistemáticas e/ou que não considerem a importância do treino de generalização, algo que implicaria no ensino de habilidades e comportamentos rígidos.

Os relatos colhidos permitem afirmar que a estimulação precoce realizada a partir da ABA, quando bem planejada e adequadamente implementada, gera melhorias significativas no repertório comportamental das crianças com TEA e, consequentemente, na qualidade de vida das famílias.

\section{Considerações finais}

A ABA é um referencial consistente e de grande importância na proposição de estimulações precoces e intensivas em crianças com TEA, com 0 intuito de minimizar comportamentos-problema e oportunizar melhorias consideráveis na vida do cliente, seja a partir da aquisição de novas habilidades, seja a partir da manutenção daquelas já existentes.

Devido ao aumento nos diagnósticos de TEA, verifica-se a importância da formação de profissionais, das diversas áreas da saúde, capacitados para a atuação multidisciplinar técnica e eticamente comprometida. Tais intervenções exigem dedicação e afeto por parte dos profissionais, sobretudo do psicólogo, de modo que este profissional seja capaz de entender as especificidades do público autista e de sua família, além de estar teórica e tecnicamente habilitado para a realização de intervenções seguras e eficazes.

A partir das informações obtidas nas entrevistas e das análises realizadas, espera-se ter contribuído para a elucidação de aspectos relativos à intervenção em casos de TEA a partir da perspectiva da ABA. Considera-se que essa abordagem tem obtido grandes avanços no tratamento de crianças com autismo, por permitir o estabelecimento de intervenções efetivas e relevantes. 


\section{Referências}

Almeida, C. G. M., \& Martone, M. C. C. Ensino por tentativas discretas para pessoas com transtorno do espectro autista. In A. C. Sella \& M. A Ribeiro. (Orgs.), Análise do Comportamento Aplicada ao Transtorno do Espectro Autista (pp. 189-203). Curitiba: Appris, 2018.

Andrade, A. A., \& Teodoro, M. L. M. (2012). Família e Autismo: Uma Revisão da Literatura. Contextos Clínicos, 5(2), p. 133-143. https://doi.org/10.4013/ ctc.2012.52.07

American Psychiatric Association - APA. (2014). Manual Diagnóstico e Estatístico de Transtornos Mentais - DSM-5. Porto Alegre: Artmed.

Banaco, R. A., Zamignani, D. R., Martone, R. C., Vermes, J. S. \& Kovac, R. (2012). Psicopatologia. In M. M. C. Hubner \& M. B. Moreira (Orgs.), Temas clássicos da psicologia sob a ótica da análise do comportamento (pp. 154-166). Rio de Janeiro: Guanabara Koogan.

Barros, R. S., Barbosa, A. A. \& Silva, A. J. M. (2018). Intervenção comportamental ao transtorno do espectro autista implementada via cuidadores. In A. C. Sella \& M. A Ribeiro. (Orgs.), Análise do Comportamento Aplicada ao Transtorno do Espectro Autista (pp. 251-260). Curitiba: Appris, 2018.

Bosa, C. A., \& Zanon, R. B. (2016). Psicodiagnóstico e transtorno do espectro autista. In C. S. Hutz, D. R. Bandeira, C. M. Trentini, \& J. S. Krug. (Orgs.), Psicodiagnóstico (pp. 308-322). Porto Alegre: Artmed.

Brasil. (2014). Diretrizes de atenção à reabilitação da pessoa com transtorno do espectro autista. Brasília: Ministério da Saúde.

Brasil. (2015) Linha de cuidado para a atenção às pessoas com transtornos do espectro do autismo e suas famílias na rede de atenção psicossocial do sistema único de saúde. Brasília: Ministério da Saúde.

Canovas, D. (2018). Escolha das medidas do comportamento. In A. C. Sella \& M. A Ribeiro. (Orgs.), Análise do Comportamento Aplicada ao Transtorno do Espectro Autista (pp. 171-188). Curitiba: Appris, 2018.

Castro-Souza, R. M. (2011). Adaptação brasileira do M-CHAT (Modified Checklist for autism in toddlers). Dissertação de Mestrado. Universidade de Brasília, Distrito Federal, Brasil.

Figueiredo, C. S. (2014). Um estudo sobre programas de intervenção precoce e o engajamento dos pais como co-terapeutas de crianças autistas. Dissertação (mestrado). Pontifícia Universidade Católica do Rio de Janeiro, Rio de Janeiro, RJ.

Gomes, P. T. M., Lima, L. H. L., Bueno, M. K. G., Araújo, L. A., \& Souza, N. M. (2015). Autism in Brazil: a systematic review of family challenges and coping strategies. Jornal de Pediatria, 91(2), 111-121. https://doi. org/10.1016/j.jped.2014.08.009

Hyman, S. L., \& Johnson, J. K. (2012). Autism and Pediatric Practice: Toward a Medical Home. J Autism Dev Disord 42, 1156-1164. https://doi. org/10.1007/s10803-012-1474-3

Lafrance, D. (2018). Planejando intervenções individualizadas. In A. C. Sella \& M. A Ribeiro. (Orgs.), Análise do Comportamento Aplicada ao Transtorno do Espectro Autista (pp. 137-166). Curitiba: Appris, 2018.

Lord, C., Rutter, M., Goode, S., Heemsbergen, J., Jordan, H., Mawhood, L. \& Schopler, E. (1989). Austism diagnostic observation schedule: A standardized observation of communicative and social behavior. Journal of Autism and Developmental Disorders, 19, 185-212. https://doi.org/10.1007/ BF02211841

Lord, C., Rutter, M., \& Le Couteur, A. (1994). Autism Diagnostic Interview-Revised: A revised version of a diagnostic interview for caregivers of individuals with possible pervasive developmental disorders. Journal of Autism and Developmental Disorders, 24(5), 659-685. https://doi. org/10.1007/BF02172145

Losapio, M. F., \& Pondé, M. P. (2008). Tradução para o português da escala M-CHAT para rastreamento precoce de autismo. Revista de Psiquiatria do Rio Grande do Sul, 30(3), 221-229. https://doi.org/10.1590/S010181082008000400011
Mapelli, L. D., Barbieri, M. C., Castro, G. V. D. Z. B., Bonelli, M. A., Wernet, M., \& Dupas, G. (2018). Child with autistic spectrum disorder: care from the family. Escola Anna Nery. Revista de Enfermagem, 22(4), 1-9, 2018. https:// doi.org/10.1590/2177-9465-EAN-2018-0116

Marques, D. F., \& Bosa, C. A. (2015). Protocolo de avaliação de crianças com autismo: Evidências de validade de critério. Psicologia: Teoria e Pesquisa, 31(1), 43-51. https://doi.org/10.1590/0102-37722015011085043051

Martone, M. C. C. (2017). Tradução e adaptação do Verbal Behavior Milestones Assessment and Placement Program (VB-MAPP) para a língua portuquesa e a efetividade do treino de habilidades comportamentais para qualificar profissionais. Tese (doutorado). Universidade Federal de São Carlos, UFSCAR, São Carlos, SP.

Mesquita, W. S., \& Pegoraro, R. F. (2013). Diagnóstico e tratamento do transtorno autístico em publicações brasileiras: revisão de literatura. Journal of the Health Sciences Institute, 31 (3), 324-329.

Miele, F. G., \& Amato, C. A. H. (2016). Transtono do espectro autista: qualidade de vida e estresse em cuidadores e/ou familiares - revisão de literatura. Cadernos de Pós-Graduação em Distúrbios do Desenvolvimento, 16(2), 89-102. https://dx.doi.org/10.5935/1809-4139.20160010

Mota, A. C. W., Vieira, M. L., \& Nuernberg, A. H. (2020). Programas de intervenções comportamentais e de desenvolvimento intensivas precoces para crianças com TEA: uma revisão de literatura. Revista Educação Especial, 33(1), 1-27. http://dx.doi.org/10.5902/1984686X41167

Murari, S. C., \& Micheletto, N. (2015). Transtorno do espectro do autismo e identificação precoce de seus sinais no contexto das Unidades Básicas de Saúde. In C. M. Melo (Org), Psicologia e análise do comportamento: saúde e processos educativos (pp. 55-64). Londrina: Universidade Estadual de Londrina.

Neto, M. B. C. (2012). Análise do comportamento: behaviorismo radical, análise experimental do comportamento e análise aplicada do comportamento. Interação em Psicologia, 6(1), 13-18. http://dx.doi.org/10.5380/ psi.v6i1.3188

Pacífico, M. C., De Paula, C. S., Namur, V. S., Lowenthal, R., Bosa, C. A., \& Teixeira, M. C. T. V. (2019). Preliminary evidence of the validity process of the Autism Diagnostic Observation Schedule (ADOS): translation, cross- cultural adaptation and semantic equivalence of the Brazilian Portuguese version. Trends in Psychiatry and Psychotherapy, 41 (3), 218-226. https:// doi.org/10.1590/2237-6089-2018-0063

Robins, D. L., Fein, D., Barton, M. L., \& Green, J. A. (2001). The Modified Checklist for Autism in Toddlers: an initial study investigating the early detection of autism and pervasive developmental disorders. Journal of Autism and Developmental Disorders, 31 (2), 131-144. https://doi. org/10.1023/A:1010738829569

Robins, D. L., Fein, D., Barton, M. L., \& Resegue, R. M. (2009). Questionário Modificado para a Triagem do Autismo em Crianças entre 16 e 30 meses, Revisado, com Entrevista de Seguimento (M-CHAT-F/F). Disponível em: https://mchatscreen.com/wp-content/uploads/2018/04/M-CHAT-R_F_Brazilian_Portugese.pdf. Acesso em 30 mar. 2021.

Ribeiro, D. M., \& Sella, A. C. (2018). Descobrindo as preferências da pessoa com transtorno do espectro autista. In A. C. Sella \& M. A Ribeiro. (Orgs.), Análise do Comportamento Aplicada ao Transtorno do Espectro Autista (pp. 105-124). Curitiba: Appris, 2018.

Ribeiro, D. M., Sella, A. C., \& Souza, A. A. (2018). Avaliação do Comportamento. In A. C. Sella \& M. A Ribeiro. (Orgs.), Análise do Comportamento Aplicada ao Transtorno do Espectro Autista (pp. 125-139). Curitiba: Appris, 2018.

Rogers, S. J., \& Dawson, G. (2010). Intervenção Precoce em Crianças com Autismo: Modelo Denver para a promoção da linguagem da aprendizagem e da socialização. Lisboa: Lindel.

Seize, M. M., \& Borsa, J. C. (2017). Instrumentos para Rastreamento de Sinais Precoces do Autismo: Revisão Sistemática. Psico-USF, 22(1), p. 161-176.

Sella, A. C., \& Ribeiro, D. M. (2018). 0 que é a Análise do Comportamento 
Aplicada. In A. C. Sella \& M. A Ribeiro. (Orgs.), Análise do Comportamento Aplicada ao Transtorno do Espectro Autista (pp. 45-58). Curitiba: Appris, 2018.

Silva, M., \& Mulick, J. A. (2009). Diagnosticando transtorno Autista: aspectos fundamentais e considerações práticas. Psicologia Ciência e Profissão, 29 (1), 116-131.

Skinner, B. F. (1957). Verbal behavior. New York: Appleton-Century-Crofts, Sociedade Brasileira de Pediatria (2019). Transtorno do Espectro Autista. Manual de Orientação do Departamento Científico de Pediatria do Desenvolvimento e Comportamento, no 05, abril de 2019. Disponível em: https://www.sbp.com.br/fileadmin/user_upload/21775c-M0_-_Transtorno_do_Espectro_do_Autismo.pdf. Acesso em 30 mar. 2021.

Sundberg, M. L. (2014).VB-MAPP: Verbal Behavior Milestones Assessment and Placement Program. Guide (2nd Ed.). Concord: AVB Press.

Warren, Z., McPheeters, M. L., Sathe, N., Foss-Feig, J. H., Glasser, A., \& Veenstra-VanderWeele, J. (2011). A systematic review of early intensive intervention for autism spectrum disorders. Pediatrics, 127(5), 1303-1311. https://doi.org/10.1542/peds.2011-0426 